\title{
SEQUÊNCIAS DE MANOBRAS ASSISTIDAS POR GRAVIDADE EM ORBITAS RESSONANTES COM A LUA
}

\author{
JORGE KENNETY S. FORMIGA ${ }^{1,2}$, ANTONIO F. B.A PRADO ${ }^{1}$. \\ 1. Instituto Nacional de Pesquisas Espaciais -INPE, Av dos Astronautas 1758, SJC-SP- \\ Brasil \\ E-mails: jkennety@yahoo.com.br, prado@dem.inpe.br
}

\begin{abstract}
This research shows a study of the dynamical behavior of a spacecraft that performs a series of close approaches with the Moon. This maneuver is also known in the literature as "Swing-By Maneuver" or "Gravity Assisted Maneuver". It is a technique to reduce the fuel expenditure in interplanetary missions by replacing maneuvers based on engines by passages near a massive body. The spacecraft moves under the gravitational attraction of those two bodies and has a negligible mass. In particular, we are looking for geometries that allow multiple close approaches without any major correction maneuvers. It means that the only maneuvers allowed are the negligible ones made to force the spacecraft to pass by the Moon (or the planets) with a specified distance from its surface. So, resonant orbits are required to obtain the series of close approaches. The main motivation for this study is the existence of several studies for missions that has the goal of studying the space around the EarthMoon system using multiple close approaches to make the spacecraft to cover a larger portion of the space without any major maneuver.
\end{abstract}

Keywords— Astrodynamics, Orbital Maneuvers, Close Approach maneuvers, space trajectories.

\begin{abstract}
Resumo - Esta pesquisa apresenta um estudo do comportamento dinâmico de um veículo espacial que realiza uma série de passagens proximas a Lua. Esta manobra é também conhecida na literatura como manobra de "swing-by" ou manobra "assistida por gravidade". É uma técnica para reduzir o combustível em missões interplanetárias, substituindo manobras baseadas em motores quando a passagem proxima é realizada por um corpo maciço. O veículo espaciale se move sob a atração gravitacional dos corpos e tem uma massa desprezível. Em particular, nós estamos procurando estudar a geometria da manobra que permite múltiplas passagens próximas sem grandes manobras de correção. Isso significa que as únicas manobras desejadas são aquelas feitas para forçar uma passagem di veículo espacial proximo a Lua (ou planetas), com uma distância específica a partir da sua superfície. Assim, as órbitas ressonantes são necessários para obter a série de aproximações. A principal motivação para este estudo é a existência de vários estudos para missões que tem o objetivo de estudar o espaço em torno do sistema Terra-Lua usando várias aproximações para fazer um veículo espacial cobrir uma parcela maior do espaço sem nenhuma grande manobra.
\end{abstract}

Palavras-chave— Astronomia dinâmica, manobra orbital, manobras assistida por gravidade, trajetória espacial.

\section{Introduction}

Em engenharia aeroespacial, dependendo do propósito da missão, a trajetória da nave pode ser controlada por várias forças físicas. Neste estudo, nos concentramos em missões que usam a manobra assistida por gravidade para atuar em parte da trajetória. É bem conhecido que, quando ocorre uma grande aproximação na vizinhança de um corpo maciço (um planeta ou um satélite natural), a sonda experimenta forças físicas que dependem da velocidade relativa entre a sonda e o planeta e também sobre a distância que separa os dois, no ponto de maior aproximação.

As manobras orbitais, sem o uso de aproximações, se utiliza de um motor que pode dispor de uma força necessária para controlar o movimento durante a manobra. Estudos considerando manobras com empuxo contínuo foi apresentado por Lawden (1953, 1954), Marec (1979), Casalino et ai. (1999), Sukhanov e Prado (2001), Gomes et al. (2008) e Gomes (2012). Já considerando idéia de uma monobra impulsiva, onde a força é suposta para ter uma magnitude infinita, foi popularmente abordado na literatura, começando com Hohmann, 1925. Mais recentemente, o conceito de captura gravitacional, também tem sido considerada, em que a perturbação de um terceiro corpo (Prado, 2003), gera uma força que pode ser usado para diminuir o consumo de combustível de um espaço de manobra. Belbruno (1990), Miller e Belbruno (1991), Yamakawa et ai. (1992), Vieira-Neto e Prado (1998), Prado (2002, 2003, 2005) e Formiga e Prado (2011, 2012) mostram essa idéia em mais detalhes.

Na primeira parte do presente trabalho, uma série de passagens próximas a Lua é usada para analisar possíveis missões e cobrir o espaço em torno do sistema Terra-Lua. O sistema dinâmico dado pela "patch conics" é usado e o movimento é considerado planar em todos os lugares. A principal motivação para esta pesquisa é baseada em algumas aplicações de aproximações que têm o objetivo de manter uma nave espacial em torno da Terra, mas fazendo várias passagens pela Lua. Uma representação geométrica pode ser visto na Figura 1, onde a nave está em órbita ao redor da Terra, executando várias aproximações com a lua. É observado que o apogeu da trajetória do veículo espacial em torno da Terra está mudando, passando pelos pontos A1, A2 e A3. A figura mostra apenas os primeiros três passagens de uma série que pode ser muito maior. A distância do perigeu também muda, dando ainda mais possibilidades para estudar diferentes áreas do espaço próximo da Terra. 


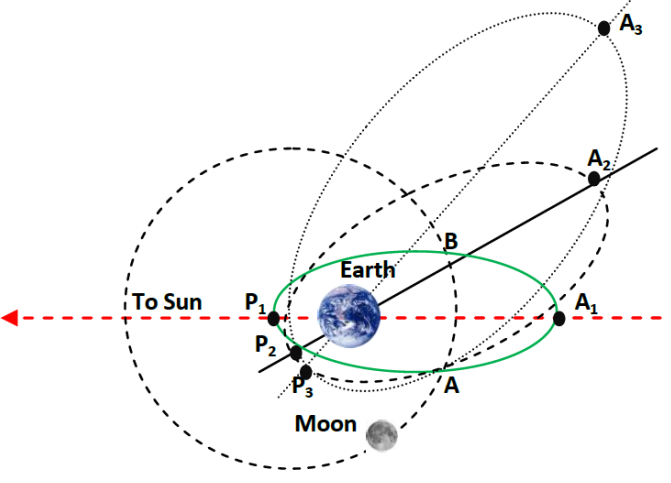

Figura 1: Visão geral de múltiplas manobras.

\section{Modelo analítico}

Este problema pode ser estudado considerando um sistema formado de três corpos: a Terra (M1), Lua (M2) e uma nave espacial com uma massa infinitesimal (M3), que permanece em órbita do Sol e, em seguida, faz uma passagem próxima com a Lua. As seguintes variáveis são utilizadas para identificar uma aproximação da trajectória: $r_{a p}$ (distância de aproximação do veículo ao centro da Lua no momento da passagem), $\vec{v}_{\infty} \infty$ (velocidades do veículo em relação a Lua, antes e após a manobra, respectivamente, no sistema inercial), $\vec{v}$ (velocidade da Lua em relação a Terra), $\delta$ (metade do ângulo de curvatura durante a apassagem próxima) e $\psi$ (Angulo de aproximação). Brouke (1988) mostra os detalhes do problema.

Os elementos orbitais $(\mathrm{a}=$ semi-eixo maior, $\mathrm{e}=$ excentricidade e), a energia (E) e momentum angular (C) da espaçonave antes do encontro com a Lua são obtidos a partir das equações: - $\quad-\quad \quad-\quad \quad-$ ),

onde $r_{p}$ é o periápsi da órbita do veículo espacial em torno da Terra, $r_{a}$ é o apoapsis dessa órbita e $\mu$ é o parâmetro gravitacional da Terra. Dessa forma, é possível obter as variações de energia e de momentum angular a partir das equações Broucke (1988):

$$
\begin{aligned}
& \infty \\
& \infty
\end{aligned}
$$

onde $\omega$ é a velocidade angular entre as primários, E-, E + é a energia antes e após a manobra, respectivamente. Finalmente, depois de ter determinado a variação da energia e quantidade de movimento angular após a manobra, é possível obter o semi-eixo maior e a excentricidade da órbita após uma manobra usando as

equações:

$$
-\mathrm{e}
$$$$
\text { 一. }
$$

Em seguida, é calculada a distância que o veículo espacial terá que passar da Lua $\left(\mathrm{r}_{\mathrm{ap}}\right)$ para atingir uma órbita que tem um valor maior para o apoapsis (assim, atingir o objetivo, fazer o veículo passar por diferentes posições no espaço), mas também que esteja em uma órbita ressonante com a órbita da Terra, de tal modo que um novo encontro irá ocorrer. Para resolver este problema, uma lista de órbitas ressonantes é feita e, em seguida, organizada de acordo com o aumento da energia. Então, o estudo consiste em encontrar o valor de $r_{\text {ap }}$ suficiente para cada passagem próxima do veículo espacial esteja na seqüência de órbitas propostas. A equação analítica para determinação desta distância segue:

$$
\frac{\mu}{\infty} \frac{}{(\delta)}
$$

onde

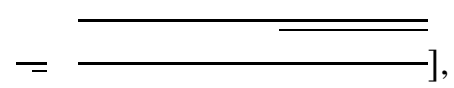

\section{Critério de Tisserand}

Critério da Tisserand foi introduzida pelo astrônomo François Felix Tisserand. Trata-se de uma equação, em coordenadas adimensionais que representa uma quantidade que deve ser conservado num sistema restrito circular dos três corpos. Assim, dois corpos que orbitam são, possivelmente o mesmo se for satisfazeita o critério do Tisserand (Battin, 1987)

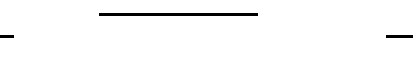

Onde $\mathrm{a}_{\mathrm{i}}, \mathrm{e}_{\mathrm{i}}$ e $\mathrm{i}_{\mathrm{i}}$ são os elementos orbitais antes da manobra e $\mathrm{a}_{0}, \mathrm{e}_{0}$ e $\mathrm{i}_{0}$ são os elementos orbitais após a manobra. $\mathrm{O}$ elementos orbitais antes e depois da manobra estão normalizados pela distância Terra-Lua. Neste estudo, o critério de Tisserand serão utilizados para validar as órbitas ressonantes encontradas.

\section{Estudo Numérico}

Nesta seção, as simulações são realizadas com o objetivo de estudar as características orbitais do veículo espacial que realiza uma série de aproximações com a Lua. Presume-se que o veículo começa seu movimento em uma determinada órbita em torno da Terra. Esta órbita é especificado pelas distâncias do seu perigeu e apogeu. $\mathrm{O}$ modelo de aproximação "patched conics" é utilizado na realização de sucessivas aproximações. Várias simulações foram feitas e escolhidas no máximo cinco voltas para a Lua entre duas passagens proximas a ela. Esta condição gera um grande número de manobras potenciais. O significado de "manobras potenciais " é que, algumas das manobras obtidos podem exigir um valor menor do que o raio da Lua, ou com uma órbita de perigeu abaixo da superfície da Terra. Assim, essas manobras deverão ser excluídas de uma lista prática de manobras. Um número superior a cinco geraria órbitas com grandes períodos, o que tem pouco interesse prático e que seria muito influenciada por outras perturbações. Estas perturbações não é considerada aqui, porque a idéia 
do presente trabalho é estudar as possibilidades e não tomar decisões finais em que órbita seria usada. A Tabela 1 mostra as órbitas, incluindo a informação do número de períodos de Lua antes da passagem próxima, o número equivalente de órbitas do veículo espacial, o período (em dias) da órbita do veículo espacial, o respectivo semimajor eixo $(\mathrm{km})$, e a ordem da ressonância.

Tabela 1. Orbitas ressonantes de um veículo espacial passando pela Lua

\begin{tabular}{|c|c|c|c|c|}
\hline $\begin{array}{l}\mathrm{N}^{\mathrm{o}} \mathrm{de} \\
\text { revoluções } \\
\text { da Lua } \\
\text { entre duas } \\
\text { passagens } \\
\text { próximas } \\
\text { sucessivas } \\
\end{array}$ & $\begin{array}{l}\mathrm{N}^{\mathrm{o}} \mathrm{de} \\
\text { revoluções } \\
\mathrm{da} \text { sonda } \\
\text { entre duas } \\
\text { passagens } \\
\text { próximas } \\
\text { sucessivas } \\
\end{array}$ & $\begin{array}{c}\text { Periodo } \\
\text { orbital da } \\
\text { sonda } \\
\text { (dias) }\end{array}$ & $\begin{array}{c}\text { Semi eixo } \\
\text { maior da } \\
\text { sonda } \\
(\mathrm{km})\end{array}$ & $\begin{array}{l}\text { Ordem da } \\
\text { ressonância }\end{array}$ \\
\hline \multirow[b]{2}{*}{1} & 1 & 27.3216 & 383182 & $1: 1$ \\
\hline & 2 & 13.6608 & 241390 & $2: 1$ \\
\hline \multirow{3}{*}{2} & 1 & 54.6432 & 608264 & $1: 2$ \\
\hline & 3 & 18.2144 & 292423 & $3: 2$ \\
\hline & 5 & 10.9286 & 208023 & $5: 2$ \\
\hline \multirow{6}{*}{3} & 1 & 81.9647 & 797052 & $1: 3$ \\
\hline & 2 & 40.9824 & 502111 & $2: 3$ \\
\hline & 4 & 20.4912 & 316310 & $4: 3$ \\
\hline & 5 & 16.3929 & 272588 & $5: 3$ \\
\hline & 7 & 11.7092 & 217815 & $7: 3$ \\
\hline & 8 & 10.2456 & 199263 & $8: 3$ \\
\hline \multirow{6}{*}{4} & 1 & 109.286 & 965559 & $1: 4$ \\
\hline & 3 & 36.4288 & 464192 & $3: 4$ \\
\hline & 5 & 21.8573 & 330217 & $5: 4$ \\
\hline & 7 & 15.6123 & 263864 & $7: 4$ \\
\hline & 9 & 12.1429 & 223160 & $9: 4$ \\
\hline & 11 & 9.93512 & 195217 & $11: 4$ \\
\hline \multirow{13}{*}{5} & 1 & 136.608 & 1120430 & $1: 5$ \\
\hline & 2 & 68.304 & 705828 & $2: 5$ \\
\hline & 3 & 45.536 & 538648 & $3: 5$ \\
\hline & 4 & 34.152 & 444644 & $4: 5$ \\
\hline & 6 & 22.768 & 339327 & $6: 5$ \\
\hline & 7 & 19.5154 & 306187 & $7: 5$ \\
\hline & 8 & 17.076 & 280108 & $8: 5$ \\
\hline & 9 & 15.1787 & 258955 & $9: 5$ \\
\hline & 11 & 12.4189 & 226529 & $11: 5$ \\
\hline & 12 & 11.384 & 213762 & $12: 5$ \\
\hline & 13 & 10.5083 & 202655 & $13: 5$ \\
\hline & 14 & 9.75771 & 192886 & $14: 5$ \\
\hline & 15 & 9.10719 & 184215 & $15: 5$ \\
\hline
\end{tabular}

Com base nesta tabela, é possível organizar estas órbitas e ter valores crescentes da energia, em seguida, procurar valores de $r_{a p}$ para cada passagem em que seja possível fazer o veículo espacial realizar esta série de manobras e finalmente analisar os elementos órbitais, energia e momento angular de acordo com a evolução das trajetórias.

A Tabela 2 representa uma sequência de manobras, em relação ao tempo, que mantém a energia crescente, semi-eixo maior e a distância do apogeu. Esta Tabela mostra as mesmas ressonancias indicadas na Tabela 1. A partir de uma dada órbita inicial em torno da Terra, o $\mathrm{r}_{\mathrm{ap}}$ é o parâmetro principal da sequiência, pois presume-se ser a única variável disponível para controlar o movimento do veículo espacial. $O$ raio da Lua é chamado $R_{m}$, assumindo o valor de $1,737 \mathrm{~km}$. A distância do perigeu da órbita inicial em torno da Terra é escolhido para ser $r_{p}$ $=9579,55 \mathrm{~km}$ e a respectiva distância apogeu é $\mathrm{r}_{\mathrm{a}}=$ $459,818.40 \mathrm{~km}$. Esta escolha é um pouco arbitrária e foi escolhida com base nos princípios de que é necessário dispor de uma órbita inicial que tem uma distância perigeu, que pode ser facilmente obtido por um padrão de propulsores, e uma distância do apogee que é um pouco acima da órbita lunar. Esses princípios geraram órbitas que podem ser alcançadas sem o consumo excessivo de combustível. Alguns testes preliminares com valores semelhantes mostraram que esse valor tem pouca influência nos resultados obtidos, uma vez que, após o primeiro encontro, a sequiência de $r_{a p}$ controla $o$ movimento do veículo espacial. Desta forma, não há necessidade de fazer muitas simulações. Agora, é necessário estudar a segunda restrição e ver se o valor para o $r_{\text {ap }}$ é assumido corretamente, o que significa que tem que ser maior que o raio da Lua. A Tabela 2 mostra que as manobras são possíveis até o número 4. Manobra 5 tem um valor de rap $=0,97$, para uma passagem por baixo da superfície da Lua, e dessa forma, não seria aplicável. Deste modo, as figuras 7 a 14 mostram os resultados para a sequência de manobras possíveis, aqueles que são numerados de 1 a 4.

Tabela 2. Sequências de manobras realizadas com passagens próximas a Lua.

\begin{tabular}{|c|c|c|c|c|c|c|c|c|c|c|c|}
\hline manobra & $\begin{array}{c}\text { Period } \\
\text { orbital } \\
\text { (day) }\end{array}$ & $\begin{array}{c}\text { rap(raios } \\
\text { da Lua) }\end{array}$ & $\mathbf{a}(\mathbf{K m})$ & e & Energia & $\mathbf{r p}(\mathbf{K m})$ & $\mathbf{r a}(\mathbf{k m})$ & $\delta\left(^{\circ}\right)$ & $\psi\left({ }^{\circ}\right)$ & resonância & $\begin{array}{l}\text { Tempo } \\
\text { (days) }\end{array}$ \\
\hline 0 & 13.097 & - & 234698.97 & 0.9592 & -0.8492 & 9579.55 & 459818.4 & 36.97 & 358.88 & - & 0 \\
\hline 1 & .6608 & 1.59 & 241389.80 & 0.9516 & -0.8256 & 11683.49 & 471096.11 & 40.55 & 357.54 & $2: 1$ & 27,32 \\
\hline 2 & 15.1787 & 1.33 & 258954.77 & 0.9321 & -0.7696 & 17584.71 & 500324.82 & 43.60 & 359.41 & $9: 5$ & 163,93 \\
\hline 3 & 15.6123 & 1.21 & 263864.03 & 0.9269 & -0.7553 & 19300.26 & 508427.80 & 45.17 & 359.03 & $7: 4$ & 273,22 \\
\hline 4 & 16.3929 & 1.09 & 272587.78 & 0.9179 & -0.7311 & 22391.13 & 522784.43 & 46.91 & 359.23 & $5: 3$ & 355,18 \\
\hline 5 & 17.0760 & 0.97 & 280108.06 & 0.9105 & -0.7115 & 25083.13 & 535132.99 & 48.81 & 358.86 & $8: 5$ & 491,79 \\
\hline 6 & 18.2144 & 0.84 & 292422.91 & 0.8991 & -0.6815 & 29510.94 & 555334.87 & 51.08 & 358.88 & $3: 2$ & 546,43 \\
\hline
\end{tabular}




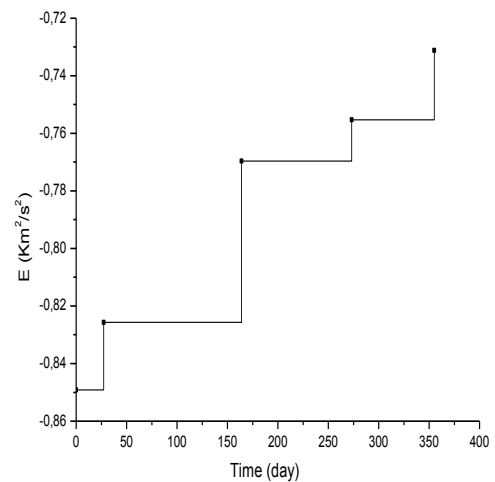

Figura 7. Energia de um veículo especial em função do tempo.

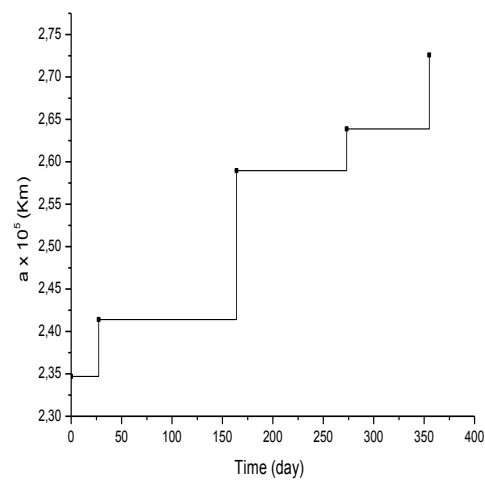

Figura 8. Semi-eixo maior de um veículo especial em função do tempo.

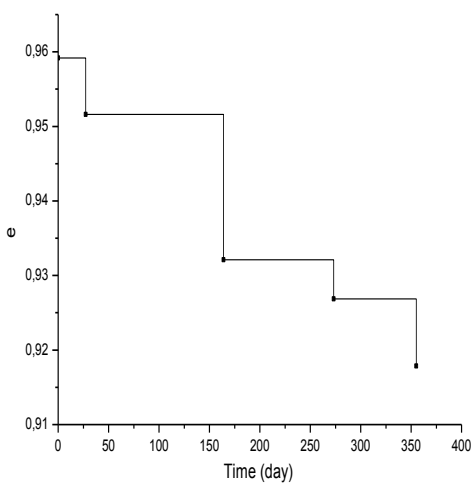

Figura 9. Excentricidade de um veículo especial em função do tempo.

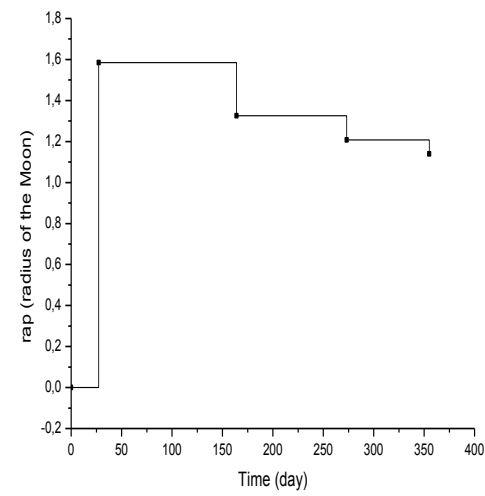

Figura 10. Distância rap de um veículo especial em função do tempo.

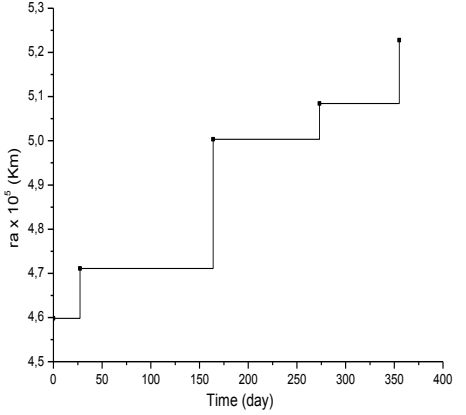

Figura 11. Distância do apogee de um veículo especial em função do tempo.

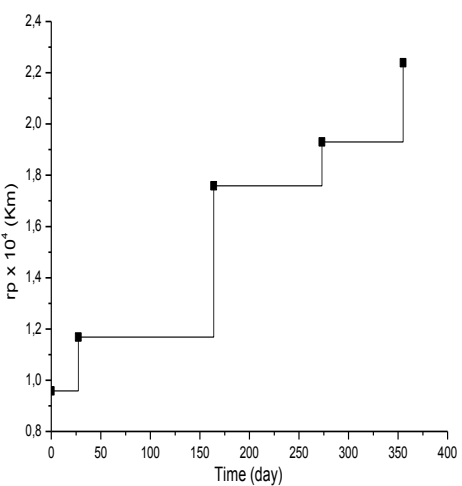

Figura 12. Distância do apogeu de um veículo especial em função do tempo.

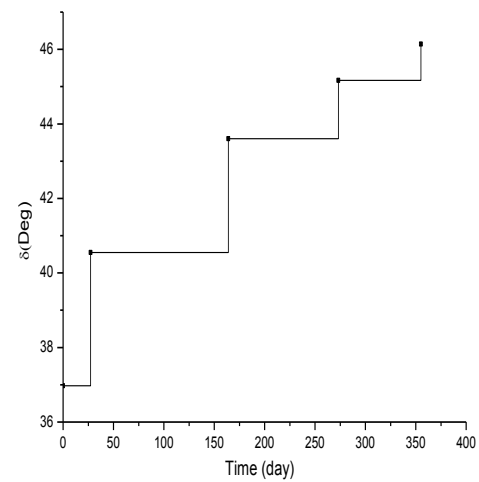

Figura 13. Ângulo de deflexão de um veículo especial em função do tempo.

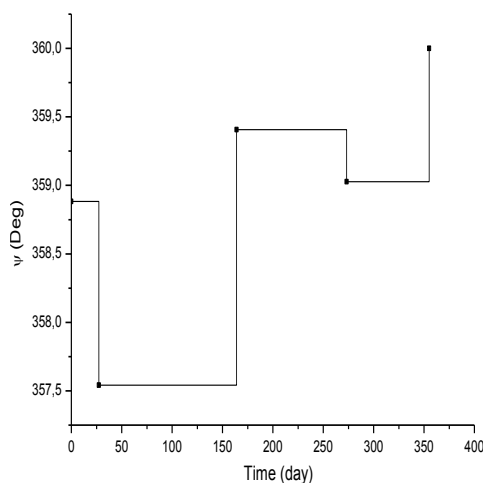

Figura 14. Ângulo de aproximação de um veículo especial em função do tempo. 
Note que o veículo não permanece em cada órbita ao mesmo tempo. O período de cada órbita são visíveis nos intervalos limitados pelos pontos nos gráficos que representam o momento de cada manobra.

Esses resultados mostram a evolução dos parâmetros com conclusões interessantes em relação a energia e assim o momentum angular (devido a Eq 2.), o semi-major (eixo) e o aumento da distância do apogeu após cada passagem próxima. Uma característica é forçada pela escolha da distância mais próxima da Lua, uma vez que o objetivo da pesquisa é encontrar uma série de manobras. A energia vai de $-0,8492 \mathrm{~km}^{2} / \mathrm{s}^{2}$, antes da primeira passagem até $-0,7311 \mathrm{~km}^{2} / \mathrm{s}^{2}$ após o última passagem possível, em etapas crescentes de valores. Esta variação faz com que a energia no semi-eixo maior aumente de 234,698.97 km para 272,587.78 km, o que corresponde a uma variação da apogeu de 459,818.40 km (um pouco acima da órbita Lunar) para $522,784.43 \mathrm{~km}$. A excentricidade mostra uma sequência de valores decrescentes de ainda divididas em pequenos passos. $\mathrm{O}$ intervalo de tempo para esta sequência é 355,18 dias, muito perto de um ano. Os valores de $r_{a p}$ tem um comportamento muito interessante. Ele reduz a cada passagem. A razão disto é a necessidade de compensar o aumento da velocidade do veículo espacial. As equações (1) e (2) mostram que a variação de energia depende da velocidade de aproximação e distância do periapsis e da passagem próxima. Os outros parâmetros são todos constantes. Assim, desde que cada passagem aumente a energia do veículo espacial e todas as passagens têm a mesma distância da Terra (a distância Terra-Lua), a velocidade devido ao encontro com a Lua também aumenta de passagem para passagem. Isto significa que a distância periapsis tem de diminuir para ser capaz de compensar a variação necessária de energia. Isto é mostrado na Figura 10 e na Tabela 2. Na verdade, esta é a razão pela qual a seqüência termina em um certo ponto antes do veículo escapar. Há um ponto (manobra 5), onde um valor está abaixo da superfície lunar o qual seria necessária uma manobra fora da orbita ressonante para se obter um aumento da energia. Figura 13, que mostra a sequência do ângulo de deflexão, confirma este resultado, mostrando que este valor sempre aumentar para compensar o aumento da velocidade de aproximação. Figura 14 complementa presente estudo, demonstrando que as passagens estão sempre em frente da Lua, para aumentar a energia.

\section{Conclusão}

Este estudo foi realizado para mostrar a evolução das trajetórias, bem como as amplitudes das variações do impulso na velocidade, energia e momento angular de uma órbita devido a uma série de aproximações com um corpo celestial, neste caso a Lua. Um conjunto de equações analíticas foram desenvolvidas para permitir o cálculo da distância de aproximação que gera uma órbita especificado. Em seguida, uma série de órbitas ressonantes com a Lua, que venha a aumentar o apoapsis para cobrir uma grande área do espaço em torno do sistema Terra-Lua é encontrado. Usando esta equação, é possível estabelecer uma sequência de aproximações que cumpre os objetivos.

Os resultados mostraram que é possível encontrar seqüências úteis de aproximações para estudar o espaço ao redor da Lua usando estas mudanças naturais de órbitas, passando por diferentes posições no espaço, sem as despesas de aplicação de um controle.

Quanto ao estudo da precisão do método de "patchcónicas" para estudar este problema, foi mostrado que a sua precisão melhora quando a distância de aproximação diminui, o que é muito importante, porque as aplicações práticas estão concentrados nesta região.

\section{Agradecimentos}

Os autores desejam expressar seus agradecimentos à CAPES (Agência Federal para a Educação de PósGraduação - Brasil), CNPq (Conselho Nacional de Desenvolvimento Científico e Tecnológico), FAPESP (Fundação de Pesquisa de São Paulo) e INPE (Instituto Nacional de Pesquisas Espaciais, Brasil ) para contribuir e apoiar a pesquisa.

\section{Referências bibliográficas}

Battin, R.H., A (1987). An Introduction to the Mmathematics and Models of Astrodynamics, AIAA, New York, New York.

Belbruno, E.A. (1990). Examples of the Nonlinear Dynamics of Ballistic Capture and Escape in the Earth-Moon System. AIAA-90-2896. In: AIAA Astrodynamics Conf., Portland, Oregon.

Broucke, R.A. (1999). The Celestial Mechanics of Gravity Assist, In:AIAA/AAS Astrodynamics Conf., Minneapolis, MN, August (AIAA paper 88-4220).

Casalino, L.; Colasurdo, G. and Pasttrone, D. (1999). Optimal Low-thrust Escape Trajectories Using Gravity Assist. Journal of Guidance, Control and Dynamics, 22(5), pp. 637-642. DOI: 10.2514/2.4451

Formiga, J. K. S. and Prado, A. F. B. A. (2011). Orbital Characteristics Due to the Three Dimensional SwingBy in the Sun-Jupiter System. In: 10 International Conference on Computational Intelligence ManMachine Systems and Cybernetics, Jakarta: The World Scientific and Engineering Academy and Society Press, vol. 1. pp. 61-69.

Formiga, J. K. S. and Prado, A. F. B. A. (2012). Analytical Three-Dimensional Study of Maneuvers Assisted by Gravity. In: 3rd European Conference of Mechanical Engineering, Paris. Procceedings of the 3rd European Conference of Mechanical Engineering. Paris: WSEAS. vol. 1. pp. 115-121.

Formiga, J. K. S. and Prado, A. F. B. A. (2012). A Close Approach Between a Planet and a Particle: Sun- 
Jupiter System. In: Proceedings of the 10th International Conference on System Science and Simulation in Engineering. Penang, Malasia: World Scientific and Engineering Academy and Society Press, vol. 1. pp. 32-37

Gomes, V.M.; Prado, A.F.B.A. and Kuga, H.K. (2008), Low Thrust Maneuvers for Artificial Satellites. Transactions on Applied and Theoretical Mechanics, vol.3, pp. 10 .

Gomes, V.M. and Prado, A.F.B.A. (2010). A Study of the impact of the initial energy in a close approach of a cloud of particles. WSEAS Transactions on Mathematics, 9, 811-820.

Gomes, V.M. and Prado, A.F.B.A. (2012). Low-thrust Out-of-plane Orbital Station-keeping Maneuvers for Matellites. Mathematical Problems in Engineering (Print), pp.1-14. DOI: 10.1155/2012/532708

Lawden, D.F..(1953). Minimal Rocket Trajectories. ARS Journal, vol. 23(6), pp.360-382.

Lawden, D.F.(1954). Fundamentals of Space Navigation. JBIS, vol.13, pp.87-101.

Marec, J.P. 1979. Optimal Space Trajectories. Elsevier, New York.

Miller, J.K. and Belbruno, E.A. (1991). A method for the Construction of a Lunar Transfer Trajectory Using Ballistic Capture. AAS-91-100. In: AAS/AIAA Space Flight Mechanics Meeting, Houston, Texas.
Prado, A.F.B.A. (2002). Numerical Study and Analytic Estimation of Forces Acting in Ballistic Gravitational Capture. Journal of Guidance Control and Dynamics, vol.25(2), pp.368-375. DOI: 10.2514/2.4891

Prado, A.F.B.A. (2003). Third-Body Perturbation in Orbits Around Natural Satellites. Journal of Guidance, Control and Dynamics, vol.26(1), pp. 3340. DOI: $10.2514 / 2.5042$

Prado, A.F.B.A. (2005). Numerical and Analytical Study of the Gravitational Capture in the Bicircular Problem. Advances in Space Research, vol.36(3), pp.578-584. DOI: 10.1016/j.asr.2004.12.033

Vieira-Neto, E. and Prado, A.F.B.A.(1998), Time-offlight Analyses for the Gravitational Capture Maneuver. Journal of Guidance, Control and Dynamics, vol.21(1), pp.122-126.

DOI: $10.2514 / 2.4207$

Sukhanov A.A. and Prado A.F.B.A.(2001). Constant Tangential Low-thrust Trajectories Near an Oblate Planet. Journal of Guidance Control and Dynamics, vol.24(4), pp.723-731. DOI: 10.2514/2.4772

Yamakawa, H., Kawagughi, J., Isgii, N. and Matsuo, H. (1992). A Numerical Study of Gravitational Capture Orbit in the Earth-Moon System. AAS 92-186. In: AAS/AIAA Spaceflight Mechanics Meeting, Colorado Springs, Colorado, pp.24-26. 